\title{
ORIGINAL
}

\section{PREVALENCIA DE HIPERLIPIDEMIA EN NIÑOS Y ADOLESCENTES DE LA PROVINCIA DE CÁCERES}

\author{
Luis Prieto Albino (1), Javier Arroyo Díez (2), José M. ${ }^{a}$ Vadillo Machota (2), Concha Mateos \\ Montero (3) y Angel Galán Rebollo (2).
}

(1) Centro de Salud «Zona Sur». Cáceres.

(2) Hospital San Pedro de Alcántara. Cáceres.

(3) Facultad de Veterinaria. Cáceres.

\section{RESUMEN}

Fundamento: Actualmente se considera al factor lipídico como el principal responsable del riesgo cardiovascular del individuo joven, factor ya presente en la infancia en proporciones preocupantes y crecientes como parecen mostrar diversos estudios nacionales. En el presente trabajo se analiza la prevalencia de hiperlipidemia en niños de la provincia de Cáceres según diversos criterios, describiendo los cambios según grupos de edad y sexo, y comparando los resultados globales con otros estudios autóctonos.

Método: Estudio descriptivo transversal de una muestra representativa y proporcional de 2.150 niños ( 2 a 16 años) de la provincia de Cáceres $(\mathrm{N}=91.083)$. Se determina: colesterol total, fracciones, apolipoproteinas y cocientes de riesgo (técnica enzimática).

Resultados: El 27.9\% de los niños presenta valores de colesterol total $200 \mathrm{mg} / \mathrm{dl}$.; $7.5 \%$ de las mujeres y $4.7 \%$ de $10 \mathrm{~s}$ varones muestran cifras superiores a $230 \mathrm{mg} / \mathrm{dl}$. Niveles de C-LDL $>130 \mathrm{mg} / \mathrm{dl}$, aparecen en un $26.4 \%$, de Apo-B $>75 \mathrm{mg} / \mathrm{dl}$ en el $65.5 \%$ y de C-NO-HDL $>165$ en el $8.4 \%$. Un C-HDL $<35$ $\mathrm{mg} / \mathrm{dl}$. se encontró en $3.3 \%$ de los individuos mientras que lá Apo-A $<100 \mathrm{mg} / \mathrm{dl}$. sólo apareció en el $0.3 \%$. Las relaciones $\mathrm{CT} / \mathrm{HDL}>3.5$ y LDL/HDL $>2.2$ se mostraron en el $36.1 \%$ y en el $39.8 \%$ respectivamente. La prevalencia de hiperlipidemia es superior en mujeres y etapas prepuberales.

Conclusiones: De forma global, e independientemente del criterio utilizado, la proporción de casos de hiperlipidemia es elevada y superior a la referida en otros estudios españoles. La prevalencia de hiperlipidemia es mayor en mujeres prepuberales, mostrándose los cocientes de riesgo más discriminativos e identificando mejor el cambio del perfil lipídico puberal.

Palabras clave: Hipercolesterolemia. Prevalencia. Epidemiología. Niños. LDL-colesterol. HDL-colesterol. Apolipoproteinas. Relación CT/HDL. Relación LDL/HDL.

Correspondencia:

Luis Prieto Albino

C/ San Ignacio 6, 2. ${ }^{\circ} \mathrm{A}$

10.002 Cáceres

Tfno: 927-21 2008

\section{ABSTRACT \\ The Prevalence of Hpyerlipidemia in Children and Adolescents in the Province of Cáceres}

Background: the lipid factor is currently considered to be the main agent responsible for cardiovascular risk in young individuals. This is already present during infancy in proportions that give rise to concern, and is growing, as has would seem to have been shown by several national studies. This study analyses the prevalence of hyperlipidemia in children within the province of Caceres according to several different criteria, and describes changes according to age groups and sex, comparing the overall results with those of other national studies.

Methods: a cross - sectional descriptive study of a representative and proportional sample of 2,150 children aged 2 to 6 years old in the province of Caceres $(\mathrm{N}=91,083)$. The following were determined: total cholesterol, fractions, apolipoproteins and risk coefficients (enzymatic technique).

Results: $27.9 \%$ of children presented total cholesterol values of $>200 \mathrm{mg} / \mathrm{dl} ; 7.5 \%$ of females and $4.7 \%$ of males had figures of more than $230 \mathrm{mg} / \mathrm{dl}$. Levels of C-LDL $>130 \mathrm{mg} / \mathrm{dl}$ appear in $26.4 \%, \mathrm{Apo}-\mathrm{B}>75 \mathrm{mg} / \mathrm{dl}$ in $65.5 \%$ and C-NO-HDL $>165$ in $8.4 \%$. C-HDL- $<35 \mathrm{mg} / \mathrm{dl}$ was found in $3.3 \%$ of individuals, while Apo-A $<100 \mathrm{mg} / \mathrm{dl}$ was only found in $0.3 \%$. The ratios between CT/HDL > 3.5 and LDL/HDL > 2.2 were found to be $36.1 \%$ and $39.8 \%$, respectively. The prevalence of hyperlipide mia is higher in females and pre-puberty stages.

Conclusions: overall, and independently of the criteria used, the proportion of cases of hyperlipidemia is high, and is above the levels cited in other Spanish studies. The prevalence of hyperlipidemia is highest in females before puberty, who display the most discriminatory risk coefficients, and best identify the change in the lipid profile at puberty.

Key words: Hypercholesterolemia. Prevalence. Epidemiology. Children. LDL-cholesterol. HDL-cholesterol. Apolipoproleins. CT/HDL ratio. LDL/HDL ratio. 


\section{INTRODUCCIÓN}

Las enfermedades cardiovasculares (ECV) constituyen la primera causa de muerte en los países desarrollados. En el año 1992 estas enfermedades ocasionaron el $40 \%$ de todas las defunciones en España, ocupando el primer lugar las de origen cerebrovascular con el $31.7 \%$, seguido de las isquémicas cardiacas con el $26.6 \%$.

Sin embargo aunque en nuestro pais, como en la mayoría de los industrializados, se observa una tendencia descendente en la mortalidad cardiovascular desde la década de los 60, esta se debe fundamentalmente a una mayor caída de la mortalidad cerebrovascular, comenzando a predominar desde el año 1987 las enfermedades coronarias en varones y a reducirse las distancias entre ambos procesos en mujeres.

Se ha demostrado que el proceso causal de estas enfermedades, la ateromatosis, sc inicia en edades tempranas ${ }^{2}$, influyendo en su evolución una serie de factores (tradicionalmente conocidos como de «riesgo cardiovascular $)^{3,4}$, alguno de ellos ya presentes en la infancia, cuyo efecto conjunto potencia el riesgo de dicha enfermedad ${ }^{5}$.

Es conocida la participación de los lípidos en la géncsis del atcroma ${ }^{6}$ y que la incidencia de ECV se relaciona directamente con los niveles de colesterol, o más propiamente con los de c-LDL ${ }^{7,8}$, siendo el factor lipídico el principal marcador de la enfermedad en individuos con menos de 55 años ${ }^{3}$. Por otro lado, el hecho de que una apreciable proporción de niños con colesterol elevado mantenga sus cifras en la edad adulta (fenómeno de Tracking o Rastreo ${ }^{9,10}$ ), la demostrada agregación familiar de las hiperlipidemias ${ }^{11}$, así como la tendencia creciente de las cifras medias de colesterol observadas en la población infantil española en las últimas décadas ${ }^{12-16}$ justifican la realización de estudios para identificar, entre la población infantil, aquellos individuos con cifras altas de lípidos para iniciar medidas preventivas contra la enfermedad.

El presente trabajo tiene como objetivo conocer la prevalencia de hiperlipidemia en niños y adolescentes de la provincia de Cáceres, analizando las variaciones de este parámetro por grupos de edad y sexo.

\section{SUJETOS Y MÉTODOS}

Durante el curso 1992-93 se estudiaron 2.150 niños y adolescentes (ambos sexos al $50 \%$ ), con edades comprendidas entre los 2 y 16 años, pertenecientes a 34 centros escolares de la provincia de Cáceres seleccionados aleatoriamente. En todos los casos se eligieron clases enteras.

Para el cálculo del tamaño muestral se consideró una prevalencia de hiperlipidemia del $35 \%$ (superior a la referida en otros estudios de características similares ${ }^{15.17-19}$ ) para un nivel de confianza del $95 \%$ y una precisión del $2 \%$ con el fin de que los resultados fueran lo suficientemente representativos de la población de referencia cifrada en 91.083 individuos según los datos más recientes al inicio del estudio (censo de 1985).

La provincia fue dividida en 8 zonas, cada una con un núcleo de referencia donde se realizó el trabajo de campo (figura 1). Debido a la concentración escolar, la mayor parte de los niños de las poblaciones circundantes acudían a estudiar a estos núcleos por lo que consideramos que la población rural está lo suficientemente representada. Igualmente, la representación de cada estrato de edad siguió las proporciones de la población escolar durante el curso 1992-93 en cada una de las 8 zonas referidas.

Se dividió a los escolares en tres grupos etáreos: de 2 a 5 , de 6 a 12 y de 13 a 16 años; a todos ellos, tras la oportuna autorización de sus familiares, se les realizó una extracción de $4 \mathrm{cc}$. de sangre desde una vena antecubital, en condiciones estandarizadas ${ }^{20} \mathrm{y}$ tras un ayuno mínimo de 12 horas. Las 
Figura 1

División de las zonas de la provincia de Cáceres y localidades donde se realizó el estudio

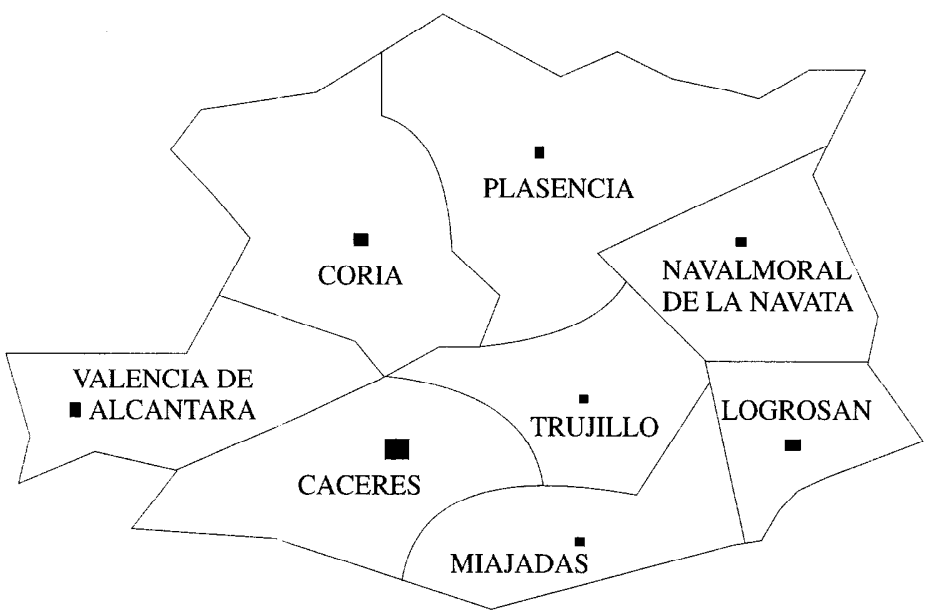

muestras fueron recogidas en tubos Vacutainer sin ningún aditivo, las cuales, tras un reposo de 30 minutos a temperatura ambiente, fueron centrifugadas in situ hasta obtener suero y seguidamente refrigeradas en nevera portátil hasta su traslado al Hospital San Pedro de Alcántara de Cáceres donde, una vez separados los sueros, estos fueron congelados $\mathrm{a}-70^{\circ}$ hasta proceder a su análisis por grupos. Este incluyó la determinación de Colesterol Total (CT), colesterol HDL (c-HDL), colesterol LDL (c-LDL), colesterol NO HDL (c-NO HDL), y apoproteinas A1 y B100.

El colesterol total se determinó mediante técnica enzimática colorimétrica por el método CHOD-PAP ${ }^{21}$ utilizando reactivos del Instituto Boehringer Mannheim con un analizador COBAS-MIRA-S. El colesterol HDL (c-HDL) se determinó, tras la precipitación de las VLDL y LDL mediante ácido fosfotúngstico y cloruro de magnesio ${ }^{22}$, con la misma metodología descrita para el colesterol total, utilizando reactivos de Boehringer Mannheim. El colesterol LDL (cLDL) se calculó mediante la fórmula de Friedewald-Fredrikson ${ }^{23}$ : c-LDL $=$ CT $-(c-$ $\mathrm{HDL}+\mathrm{TG} / 5)$. El c-NO HDL se obtuvo restando el c-HDL del CT (c-NO-HDL =
$=\mathrm{CT}-\mathrm{c}-\mathrm{HDL})$. Finalmente, la determinación de las apoproteinas se realizó mediante ensayo inmunoturbidimétrico ${ }^{24}$ utilizando antisueros del Instituto Boehringer Mannheim.

Todas las determinaciones fueron sometidas a un control de calidad interno diario y a uno externo mensual organizado por la Sociedad Española de Química Clínica; en todos los casos, tanto los coeficientes de variación como los intervalos de desviación estándar se mantuvieron dentro de los límites permitidos y con resultados óptimos.

Se definió como riesgo lipídico $(\mathrm{mg} / \mathrm{dl})$ : $\mathrm{CT}>200, \mathrm{LDL}>130, \mathrm{HDL}<35^{25}$, c-NO HDL $>165^{26,27}$, Apo A $1<100$, Apo $\mathrm{B}>75^{17,19}$ y las relaciones CT/HDL $>3.5$ y LDL/HDL $>2.2^{17.28}$.

Se excluyeron del estudio: a) a todos los sujetos afectados en el momento del examen por patologías de carácter agudo en las tres semanas previas al estudio, b) los individuos con patología crónica, particularmente de carácter constitucional o endocrino-metabólica, y aquellos que recibían medicación que pudieran influir en las variables del estudio, c) todos aquellos que no entraron en el rango de edad de 2 a 16 años. 
El análisis estadístico de los resultados se realizó mediente el programa Statview II utilizando la prueba de $C h i^{2}$ para comparar la prevalencia de hiperlipidemia en los diferentes grupos de edad y sexo. Todos los análisis ${ }^{29}$ han sido realizados a dos colas y se ha considerado como nivel de significación el de $\mathrm{p}<0.05$.

\section{RESULTADOS}

I a tasa de respuesta fue suficiente en todos los casos (cercana al 100\%) para cubrir las necesidades del estudio con la excepción de un área de las señaladas donde fue necesario ampliar la muestra en dos centros escolares más en el grupo de niños de menor edad. En la figura 2 se representa el número total de niños y niñas (ambos sexos al 50\%) que intervinieron en el estudio. La tabla 1 muestra los valores medios de los parámetros lipídicos analizados en cada grupo de edad.

La prevalencia global de hipercolesterolemia en nuestro estudio $(\mathrm{CT}>200 \mathrm{mg} / \mathrm{dl})$ fue del $27.9 \%$, predominando esta en el sexo femenino ( 29.67 frente a un $26.14 \%$ ) si bien las diferencias no se mostraron sig- nificativas; sin embargo, al analizar un dintel más elevado (CT>230 mg/dl) la prevalencia fue claramente superior en mujeres con un $7.53 \%$ frente a un $4.74 \%$ en varoncs $(p<0.01)$. En la figura 3 se muestra la proporción de niños con cifras de CT inferiores a $175 \mathrm{mg} / \mathrm{dl}$., aquellos con niveles entre 175 200 y 201-230 así como los que superan los $230 \mathrm{mg} / \mathrm{dl}$; la figura 4 ofrece los mismos resultados según el sexo. La proporción de casos para un CT>230 mg/dl. según los diferentes grupos de edad y sexo se recoge en la tabla 2, apreciándose una prevalencia superior en mujeres, particularmente en el grupo de 13 a 16 años respecto a los varones de la misma edad.

Un C-HDL inferior a $35 \mathrm{mg} / \mathrm{dl}$ se observó en 71 individuos $(3.3 \%)$, no apareciendo diferencias significativas entre sexos por grupos de edad, aun cuando esta alteración se mostró más prevalente en el grupo de mujeres de 2 a 5 años $(7.4 \%$ frente a $5.2 \%$ ) y de varones adolescentes $(6.64 \%$ frente a $3.75 \%$ ), resultados que se muestran en la tabla 2. La proporción de casos con Apo$\mathrm{A}<100 \mathrm{mg} / \mathrm{dl}$. fue sólamente del $0.3 \%$ lo cual no permite comparaciones entre grupos con este criterio (datos no mostrados).

Figura 2

Distrihución de la muestra según edad (ambos sexos)

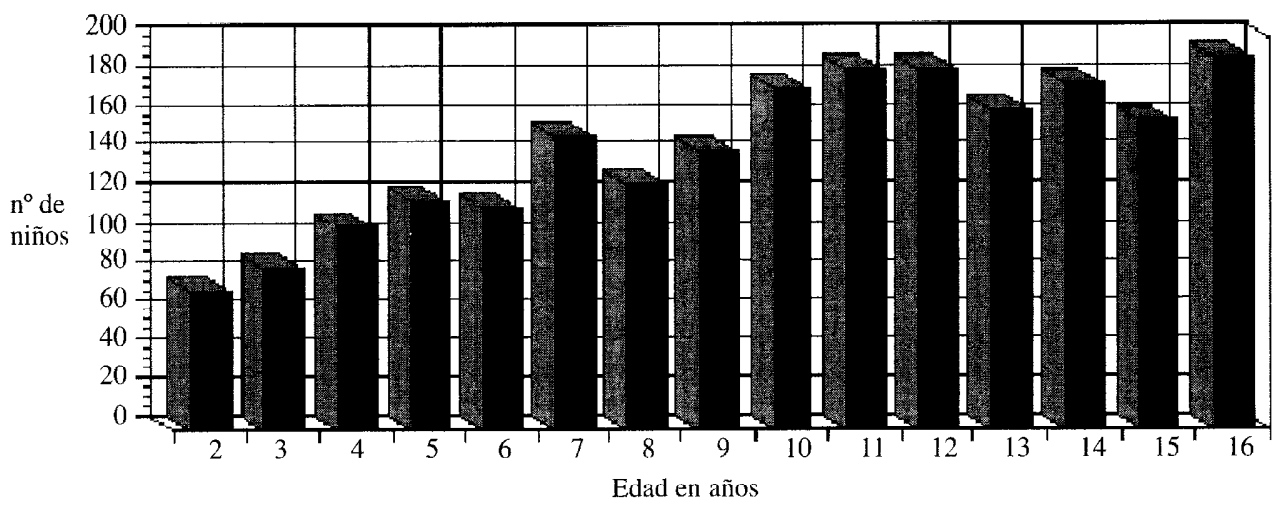


Tabla 1

Media y Desviación Estándar (DE) de los parámetros analizados en cada grupo de edad y sexo.

\begin{tabular}{|c|c|c|c|c|c|}
\hline & & \multicolumn{2}{|c|}{ VARONES } & \multicolumn{2}{|c|}{ MUJERES } \\
\hline & Años & Media & $D E$ & Media & $D E$ \\
\hline \multirow{4}{*}{$\begin{array}{l}\boldsymbol{C T} \\
\mathrm{mg} / \mathrm{dl}\end{array}$} & 2 a 5 & 185.9 & 24.9 & 186 & 28 \\
\hline & 6 a 12 & 184.6 & 28.8 & 187.8 & 29.5 \\
\hline & 13 a 16 & 171.1 & 27.7 & 180 & 28.7 \\
\hline & $2 a 16$ & 180.53 & 28.57 & 185.01 & 29.18 \\
\hline \multirow{4}{*}{$\begin{array}{l}H D L \\
\mathrm{mg} / \mathrm{dl}\end{array}$} & 2 a 5 & 55.4 & 14.7 & 51.18 & 12.07 \\
\hline & 6 a 12 & 63 & 14.6 & 59.62 & 13.03 \\
\hline & 13 a 16 & 50.5 & 11.8 & 55.86 & 12.06 \\
\hline & $2 a 16$ & 57.7 & 14.94 & 56.92 & 12.93 \\
\hline \multirow{4}{*}{$\begin{array}{l}L D L \\
\mathrm{mg} / \mathrm{dl}\end{array}$} & 2 a 5 & 119.6 & 24.3 & 123.7 & 25.8 \\
\hline & 6 a 12 & 111.2 & 26.9 & 116.5 & 26.5 \\
\hline & 13 a 16 & 109 & 25.7 & 112.1 & 26.1 \\
\hline & 2 a 16 & 112 & 26.37 & 116.38 & 26.54 \\
\hline \multirow{4}{*}{$\begin{array}{l}\text { Apo-B } \\
\mathrm{mg} / \mathrm{dl}\end{array}$} & 2 a 5 & 89.01 & 15.45 & 91.71 & 16.39 \\
\hline & 6 a 12 & 80.61 & 15.85 & 84.16 & 15.38 \\
\hline & 13 a 16 & 79.36 & 16.29 & 80.73 & 15.41 \\
\hline & $2 a 16$ & 81.68 & 16.27 & 84.38 & 15.99 \\
\hline \multirow{4}{*}{$\begin{array}{l}\text { C-NO-HDL } \\
\mathrm{mg} / \mathrm{dl}\end{array}$} & 2 a 5 & 130.55 & 25.81 & 134.83 & 26.99 \\
\hline & 6 a 12 & 121.54 & 27.87 & 128.22 & 27.55 \\
\hline & 13 a 16 & 120.63 & 27.04 & 124.17 & 27.62 \\
\hline & 2 a 16 & 122.83 & 27.46 & 128.08 & 27.69 \\
\hline \multirow{4}{*}{$\mathrm{CT} / \mathrm{HDL}$} & 2 a 5 & 3.56 & 0.99 & 3.8 & 0.96 \\
\hline & 6 a 12 & 3.04 & 0.74 & 3.26 & 0.73 \\
\hline & 13 a 16 & 3.54 & 0.92 & 3.34 & 0.82 \\
\hline & $2 a 16$ & 3.29 & 0.88 & 3.38 & 0.83 \\
\hline \multirow{4}{*}{ LDL/HDL } & 2 a 5 & 2.34 & 0.89 & 2.56 & 0.87 \\
\hline & 6 a 12 & 1.87 & 0.67 & 2.05 & 0.67 \\
\hline & 13 a 16 & 2.29 & 0.84 & 2.11 & 0.75 \\
\hline & $2 a 16$ & 2.09 & 0.8 & 2.16 & 0.76 \\
\hline
\end{tabular}

CT: Colesterol Total: HDL: Colesterol de lipoproteinas de alta densidad; LDL: Colesterol de lipoproteínas de baja densidad; Apo-B: Apolipoproteína B; C-NO-HDL: Colesterol no HDL: CT/HDL: Relación (olesterol Total/Colesterol HDL; LDL/HDL: Relación Colesterol LDL /Colesterol HDL.

Valores de C-LDL superiores a 130 $\mathrm{mg} / \mathrm{dl}$. se encontraron en 569 individuos $(26.46 \%)$ siendo más frecuente en las mujeres con un $28.93 \%$ frente a un $24 \%$ en varones $(\mathrm{p}<0.05)$. La diferente prevalencia en función de los grupos de edad y sexo se recoge en la tabla 2 donde se observa un mayor predominio en el sexo femenino, particularmente en las etapas prepuberales.

La proporción de niños con Apo-B $>75$ $\mathrm{mg} / \mathrm{dl}$. fue del $65.5 \%$, esta alteración fue más prevalente $(\mathrm{p}<0.001)$ en mujeres $(69.3 \%$ frente a $61.7 \%$ en varones). Valores de $\mathrm{C}-\mathrm{NO}-\mathrm{HDL}>165 \mathrm{mg} / \mathrm{dl}$. se presentaron en 182 niños $(8.46 \%$ del total) siendo también más frecuente $(\mathrm{p}<0.05)$ en las mujeres $(9.86 \%)$ que en los varones $(7 \%)$. Tanto en este caso como en el anterior, la proporción de niños hiperlipémicos mostró una distribución muy próxima a la ya referida para el C-LDL, es decir, mayor número de casos en el sexo femenino y principalmente en los grupos de niñas de menor edad (tabla 2). 
Figura 3

Prevalencia global de hipercolesterolemia en ambos sexos (mg./dl.)

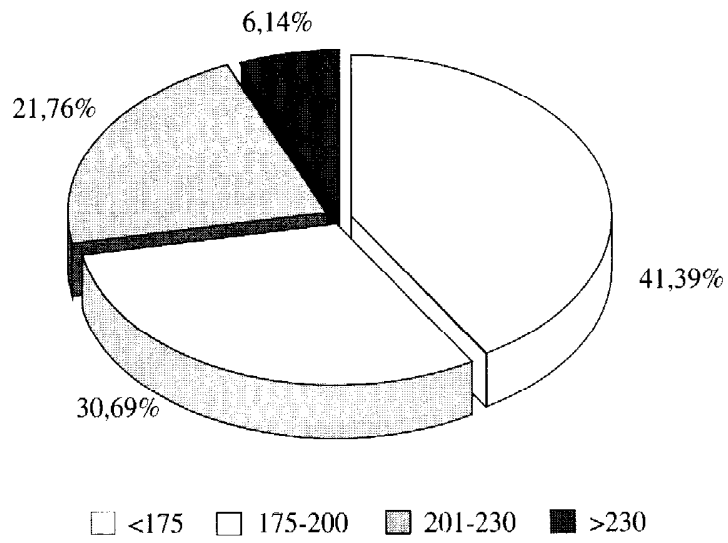

Tabla 2

Prevalencia de hiperlipidemia. Comparación entre sexos por grupo de edad

\begin{tabular}{|c|c|c|c|c|c|c|}
\hline Criterio (1) & $A \tilde{n} o s$ & $n$ & $\%$ & $n$ & $\%$ & $p$ \\
\hline \multirow{4}{*}{$C T>230$} & 2 a 5 & 7 & 3.7 & 15 & 7.93 & NS \\
\hline & 6 a 12 & 37 & 6.85 & 48 & 8.88 & NS \\
\hline & 13 a 16 & 7 & 2.02 & 18 & 5.2 & $*$ \\
\hline & $2 a 16$ & 51 & 4.74 & 81 & 7.53 & $* *$ \\
\hline \multirow{4}{*}{$H D L<35$} & 2 a 5 & 10 & 5.29 & 14 & 7.4 & NS \\
\hline & 6 a 12 & 3 & 0.55 & 8 & 1.48 & NS \\
\hline & 13 a 16 & 23 & 6.64 & 13 & 3.75 & NS \\
\hline & $2 a 16$ & 36 & 3.35 & 35 & 3.25 & NS \\
\hline \multirow{4}{*}{$L D L>130$} & 2 a 5 & 63 & 33.33 & 73 & 38.62 & NS \\
\hline & 6 a 12 & 124 & 22.96 & 157 & 29.07 & $*$ \\
\hline & 13 a 16 & 71 & 20.52 & 81 & 23.41 & NS \\
\hline & 2 a 16 & 258 & 24 & 311 & 28.93 & $*$ \\
\hline \multirow{4}{*}{$A P O-B>75$} & 2 a 5 & 150 & 79.36 & 162 & 85.71 & NS \\
\hline & 6 a 12 & 312 & 57.77 & 375 & 69.44 & $* * *$ \\
\hline & 13 a 16 & 202 & 58.38 & 209 & 60.40 & NS \\
\hline & $2 a 16$ & 664 & 61.76 & 746 & 69.39 & $* * *$ \\
\hline \multirow{4}{*}{ C-NO-HDL $>165$} & 2 a 5 & 17 & 8.99 & 27 & 14.28 & NS \\
\hline & 6 a 12 & 35 & 6.48 & 52 & 9.63 & NS \\
\hline & 13 a 16 & 24 & 6.93 & 27 & 7.8 & NS \\
\hline & $2 a 16$ & 76 & 7.06 & 106 & 9.86 & $*$ \\
\hline
\end{tabular}

(1) En mg/dl. NS: diferencias no significativas; *: $\mathrm{p}<0.05 ; * *$ : $\mathrm{p}<0.01$; ***: $\mathrm{p}<0.001$. 
Figura 4

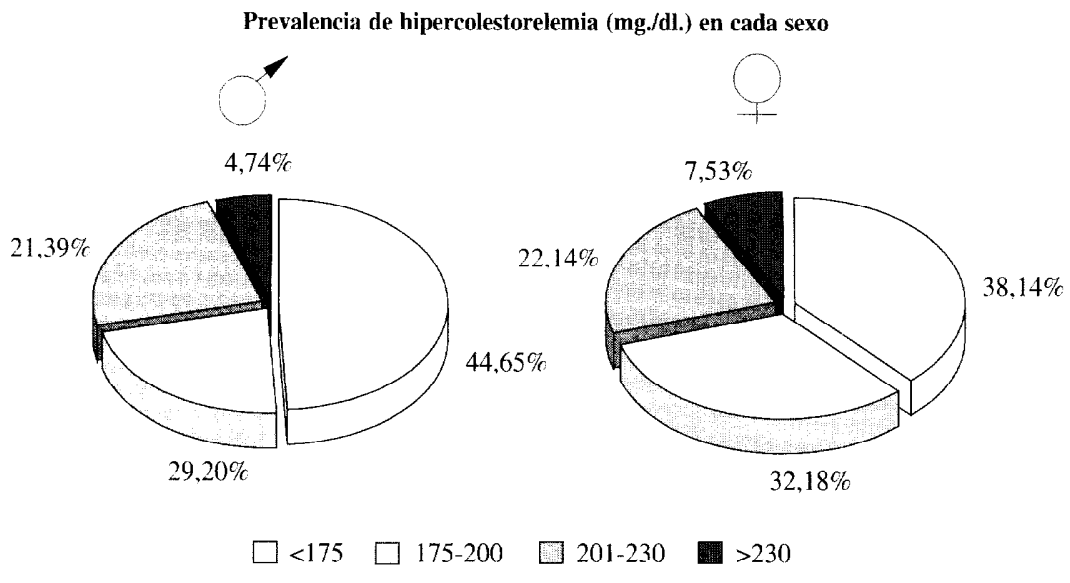

La tabla 3 muestra el porcentaje de casos con un índice CT/C-HDL $>3.5$; este fue del $36.18 \%$ predominando en mujeres con el $37.39 \%$ frente a un $34.97 \%$ en varones (diferencias no significativas). La misma tabla refleja la diferente prevalencia en función de los grupos de edad y sexo: en etapas prepuberales, y particularmente entre los 6 y 12 años, la proporción de casos es significativamente superior en las niñas, sin embargo, a partir de la adolescencia (grupo de 13 a 16 años) son los varones quienes muestran una prevalencia claramente superior $(46.24 \%$ frente a $35.26 \%)$. De todos los grupos considerados, son las mujeres de 2 a 5 años las que muestran significativamente mayor número de casos con el índice referido elevado $(59.25 \%)$.

Globalmente, un $39.86 \%$ de nuestros niños y jóvenes presentó una relación LDL/HDL superior a 2.2 (39.16\% de los varones y $40.55 \%$ de las mujeres). Al igual que en el caso anterior, la proporción de casos fue superior en las mujeres prepuberales respecto a los varones de la misma edad, invirtiéndose las proporciones a partir de los 13 años. También en este caso las mujeres de 2 a 5 años mostraron una prevalencia superior $(64.02 \%)$ al resto de los grupos (tabla 3). En las figuras 5 y 6 se compara la prevalencia de hiperlipidemia

Tabla 3

Prevalencia de hiperlipidemia según Indices de Riesgo. Comparación entre sexos por grupos de edad

\begin{tabular}{|c|c|c|c|c|c|c|}
\hline Criterio & Años & $n$ & $\%$ & $N$ & $\%$ & $p$ \\
\hline \multirow{4}{*}{$C T / H D L$} & 2 a 5 & 96 & 50.79 & 112 & 59.25 & NS \\
\hline & 6 a 12 & 120 & 22.22 & 168 & 31.11 & $* *$ \\
\hline & 13 a 16 & 160 & 46.24 & 122 & 35.26 & $* *$ \\
\hline & $2 a 16$ & 376 & 34.97 & 402 & 37.39 & NS \\
\hline \multirow{4}{*}{$L D L / H D L$} & 2 a 5 & 105 & 55.55 & 121 & 64.02 & NS \\
\hline & 6 a 12 & 147 & 27.22 & 185 & 34.25 & $*$ \\
\hline & 13 a 16 & 169 & 48.84 & 130 & 37.57 & $* *$ \\
\hline & $2 a 16$ & 421 & 39.16 & 436 & 40.55 & NS \\
\hline
\end{tabular}

NS: diferencias no significativas: *: $p<0.05 ; *^{* *}: \mathrm{p}<0.01$ 
Figura 5

Comparación de la prevalencia de hiperlipidemia según la relación CT/HDL $>3.5$ entre etapas pre y postpuberales

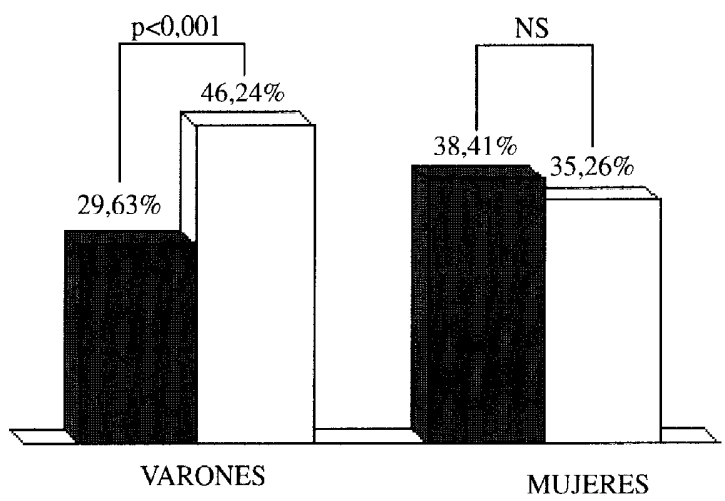

2 a 12 años 13 a 16 años

Figura 6

Comparación de la prevalencia de hiperlipidemia según la relación $L D L / H D L>2.2$ entre etapas pre y postpuberales

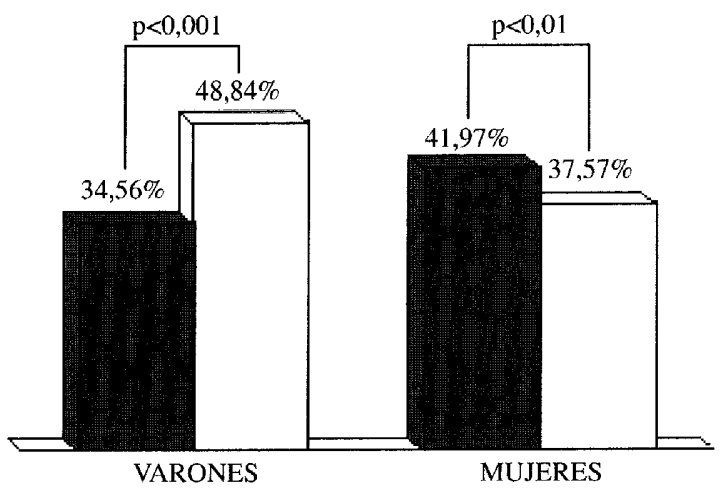

2 a 12 años 13 a 16 años

entre las etapas de 2 a 12 y 13 a 16 años (niños y niñas) en función de los índices de riesgo observándose en ambos sexos una inversión significativa para ambos parámetros a partir de la pubertad.

\section{DISCUSIÓN}

El riesgo lipídico en la infancia podría definirse a través de valores percentilados según la edad y el sexo o bien mediantc 
valores absolutos de CT, LDL o cocientes de riesgo. El empleo de los primeros se considera la forma más idónea ${ }^{30}$ al eliminar el posible error de considerar como normales un mismo valor de lípidos séricos en todas las edades y sexos, dadas las variaciones de lípidos y lipoproteínas en función de estas variables.

Respecto al colesterol, se considera tradicionalmente que no debe sobrepasar el percentil 95 aunque éste no responda necesariamente a los niveles óptimos de lípidos y lipoproteínas ${ }^{18}$, siendo más correcto utilizar tablas de C-LDL edad o bien de cocientes de riesgo-edad (CT/HDL, LDL/HDL), dado que poblaciones diferentes pueden tener niveles de C-HDL distintas y podría diagnosticarse una hipercolesterolemia a niños con niveles de HDL elevados.

Sin embargo, si se trabaja en una población como la extremeña, con alta morbimortalidad por ECV ${ }^{31}$ y con niveles de lípidos séricos generalmente elevados ${ }^{32}$, al definir el riesgo estadísticamente para aquellos valores por encima del percentil 95, se estarían considerando como normales valores muy elevados, sólo por ser muy frecuentes en dicha población. Por lo tanto, se podrían utilizar tablas percentiladas siempre que se definiera previamente por encima de que percentil se considera el riesgo.

Existe una gran diversidad de opiniones a la hora de definir cuales son los niveles absolutos de riesgo en la infancia, no obstante, la mayoría de los autore ${ }^{33}$ considera alto el límite de $200 \mathrm{mg} / \mathrm{dl}$; sin embargo, aunque la fijación es arbitraria, es necesario utilizar un rango superior de referencia para poder comparar los diversos estudios epidemiológicos infantiles. En nuestro estudio se definió el riesgo según los criterios del «Consensus Development Conference on Lowering Blood Cholesterol-1984», para el Colesterol Total, C-HDL y C-LDL ${ }^{25}$ : CT> $>200 \mathrm{mg} / \mathrm{dl}, \mathrm{C}-\mathrm{HDL}<35 \mathrm{mg} / \mathrm{dl}$ y $\mathrm{C}-\mathrm{LDL}>$ $>130 \mathrm{mg} / \mathrm{dl}$.
En el presente trabajo, la prevalencia global de hipercolesterolemia (CT>200 mg/dl) fue del $27.9 \%$, predominando en mujeres (29.67 frente a un 26.14\%) aunque las diferencias sólo se mostraron significativas al considerar un nivel de CT superior a 230 $\mathrm{mg} / \mathrm{dl}$, siendo entonces de un $7.53 \%$ en el sexo femenino frente a un $4.74 \%$ en varones $(\mathrm{p}<0.01)$. Es llamativo el hecho de que un $58 \%$ de nuestra población infantil presente cifras superiores a $175 \mathrm{mg}$., dintel a partir del cual se recomienda el consejo dietético ${ }^{34}$.

En otros estudios nacionales la prevalencia comunicada para un CT>200 $\mathrm{mg} / \mathrm{dl}$. es inferior a la nuestra: así, en Fuenlabrada ${ }^{18}$ fue de un $14 \%$, Sánchez Bayle ${ }^{19}$ encuentra un $15 \%$ en población madrileña, en el estudio de Tarancón ${ }^{17}$ fue de un $19,7 \%$, en $\mathrm{Na}$ varra $^{35}$ un $21 \%$ y en Aragón ${ }^{15}$ el $24 \%$.

Analizando los diferentes grupos de edad y sexo observamos que la prevalencia de hipercolesterolemia (>200 ó > $230 \mathrm{mg} / \mathrm{dl}$.) es superior en la mujeres prepuberales respecto a los varones de la misma edad, aunque la diferencia no se muestra significativa. A partir de la pubertad la prevalencia disminuye significativamente en ambos sexos pero en este caso se mantiene claramente superior en el sexo femenino.

$\mathrm{Al}$ analizar la prevalencia de hiperlipidemia según las cifras de LDL (>130 md/dl.) los resultados fueron similares: mayor número de casos en las etapas prepuberales -circunstancia ya referida en otros estudios y probablemente relacionada con el peculiar patrón dietético de estas edades ${ }^{36,37}-\mathrm{y}$, en cuanto al sexo, predominio en el grupo femenino, aunque en este caso las diferencias fueron significativas entre los 6 y 12 años.

La prevalencia global de HDL baja $(<35$ $\mathrm{mg} / \mathrm{dl}$.) fue de un $3.3 \%$, proporción muy superior a la encontrada en otros estudios como el de Madrid ${ }^{19}$, con un $1.84 \%$, o el de Tarancón ${ }^{17}$, con un $1.13 \%$. Esta alteración se mostró significativamente superior en el grupo de varones adolescentes $(6.64 \%)$ respecto a los niños de edades inferiores $(1.78 \%)$-si- 
tuación ya descrita en la literatura ${ }^{36}$ y posiblemente relacionada con las modificaciones hormonales de la pubertad -no apareciendo diferencias entre varones y mujeres de 13 a 16 años a pesar de ser menor el número de casos en estas últimas $(3.75 \%)$.

Habitualmente los valores de C-LDL se obtienen aplicando la fórmula de Friedewald $^{23}$, sin embargo, dicha fórmula tiene una serie de limitaciones e inconvenientes: requiere altos grados de precisión y exactitud en los elementos que la componen, considera rígida la relación entre la concentración de triglicéridos y la de C-VLDL y no es aplicable, a pesar de diversas modificaciones, cuando las cifras de triglicéridos superan los $300 \mathrm{mg} / \mathrm{dl}^{38}$. Por otro lado, las variaciones intrapersonales de los componentes de la fórmula pueden ocasionar oscilaciones superiores al $20 \%$ en el valor del C-LDL con ella obtenido.

Una solución parcial a las mencionadas limitaciones de la ecuación de Friedewald para el cálculo del C-LDL podría conseguirse con la separación de las VLDL por ultracentrifugación ${ }^{39}$ pero tiene el inconveniente de precisar una ultracentrífuga, no siempre disponible en nuestros laboratorios, por lo que se ha propuesto como alternativa determinar el colesterol NO-HDL ${ }^{49}$, restando del CT el valor obtenido para el C-HDL después de precipitar el resto de las lipoproteínas, considerando altos valores superiores a $165 \mathrm{mg} / \mathrm{dl}$. para este parámetro según las recomendaciones de las Sociedades Europea $^{26}$ y Española de Arteriosclerosis ${ }^{27}$.

Otra posibilidad sería la determinación de la apolipoproteína $B$, única apoproteína que aparece en cantidades apreciables en la LDL, la cual reúne una serie de ventajas como su alto grado de correlación con el colesterol NO-HDL y C-LDL, muy semejantes entre $\mathrm{si}^{41}$, el haberse sugerido como un predictor cardiovascular más adecuado que la $\mathrm{LDL}^{42}$, e incluso la posibilidad de monitorizar los tratamientos dietético y farmacológico a través de sus modificacio- nes ${ }^{43}$. Por otro lado, se ha descrito que la determinación de Apo B resulta más sensible y específica que la de CT para detectar niveles elevados de $\mathrm{LDL}^{44}$ siendo deseable su utilización en programas de detección infantil de hipercolesterolemia familiar ${ }^{45}$ al no precisar su determinación un ayuno previo de doce horas, si bien, la falta de valores de referencia definitivos limitan, por el momento, la utilización generalizada de este parámetro.

En nuestro estudio, tanto el colesterol noHDL como la Apo B por encima de los límites de riesgo se mostraron más prevalentes en el sexo femenino y en edades prepuberales, distribución paralela a la del C-LDL $>130$, sin embargo, la proporción de casos con el primer parámetro (colesterol NO HDL) es mucho menor (probablemente por ser más restrictivo al considerar el riesgo también en función de la HDL) y no detecta difcrencias intergrupos como en el caso de la LDL o la Apo-B. Sin embargo, como ya se ha comentado antes, parece más adecuado utilizar aquellos criterios de riesgo lipídico que tienen en cuenta las cifras de C-HDL con el fin de no sobreestimar la prevalencia de hiperlipidemia, es por ello que diversos autores proponen el empleo de cocientes de riesgo incluso en la infancia.

Basándose fundamentalmente en los resultados del estudio de Framinghan algunos autores indican que los cocientes CT/HDL y LDL/HDL tienen igual o mayor predicción, respecto al riesgo cardiovascular, que el $\mathrm{CT}$, HDL o LDL por separado ${ }^{46-48}$. Kannel $^{49}$ ha propuesto el uso del cociente CT/HDL para evaluar el riesgo, evitando así la determinación de los TG y, por tanto, la necesidad de estar en ayunas para el cálculo del C-LDL.

Paralelamente, el segundo informe del $\mathrm{NCEP}^{50}$ considera que las cifras de HDL superiores a $60 \mathrm{mg} / \mathrm{dl}$ constituyen un factor de protección contra el riesgo de presentar cardiopatía coronaria, recomendando incluir su determinación en el examen inicial, junto a 
la del CT; conocidos ambos parámetros, se infiere el cociente.

Por otro lado, en el estudio de Navarra ${ }^{28}$ se apreció que el $53 \%$ de los niños hiperlipémicos según el cociente LDL/HDL (el percentil 85 para cada grupo de edad y sexo o 2.2) presentaba cifras de $\mathrm{CT}<200 \mathrm{mg} / \mathrm{dl}$, considerando sus autores que la mejor definición de hiperlipidemia debería reducir el dintel crítico de colesterolemia (percentil 70 para cada grupo de edad y sexo) y calcular dicho cociente en los niños así seleccionados.

La bibliografía es escasa en cuento a los valores absolutos de los cocientes de riesgo por encima de los cuales se consideran estos patológicos; el NCEP ${ }^{51}$ define los valores $\mathrm{CT} / \mathrm{HDL}>4$ y LDL/HDL $>3$ para le edad adulta, sin embargo no encontramos datos referentes a la edad pediátrica en la literatura revisada.

Puesto que los estudios en población infantil española ${ }^{17,28.52}$ utilizan como criterios CT/HDL>3.5 y LDL/HDL > 2.2 también adoptamos estos límites en nuestro trabajo con el fin de comparar nuestros resultados.

Definiendo la hiperlipidemia en función de dichos cocientes de riesgo (CT/HDL $>3.5$ y LDL/HDL $>2.2$ ) la prevalencia fue de 36.18 y $39.86 \%$ respectivamente; en otros estudios como el de Navarra ${ }^{53}$ con un 14.51 y $15.7 \%$, o el de Tarancón ${ }^{17}$ con un 21.8 y $24.16 \%$ respectivamente, la prevalencia fue inferior. Según el primer cociente, la prevalencia es muy superior en mujeres durante la infancia (38\% frente a 29), invirtiéndose la relación al estudiar el grupo de adolescentes (35\% frente a 46 en varones). Según el grupo de edad, los varones adolescentes muestran una prevalencia muy superior respecto a edades inferiores; en las mujeres ocurre lo contrario, si bien las diferencias no son significativas en este caso.

Considerando el criterio LDL/HDL $>2.2$ como riesgo lipídico se observan idénticos resultados respecto al sexo y grupos de edad, esta vez con diferencias significativas en ambas etapas.

A modo de conclusión, encontramos una elevada prevalencia de alteraciones lipídicas en nuestros niños, superior a otros estudios nacionales previos, resultados coherentes con los obtenidos en población adulta extremeña ${ }^{32} \sin$ olvidar que la primera causa de mortalidad en nuestra región, las ECV, suponen un $46.78 \%{ }^{53}$ a pesar de su tendencia descendente ${ }^{54}$. Todo ello parece apuntar la necesidad de introducir modificaciones dietéticas en edades tempranas y el screening precoz de las hiperlipidemias (ya sea oportunista o selectivo) así como el de otros factores de riesgo cardiovascular, promocionando «estilos de vida» saludables, medidas perfectamente accesibles en nuestras consultas de Atención Primaria.

\section{BIBLIOGRAFÍA}

1. Instituto Nacional de Estadística. Defunciones según la Causa de Muerte 1992. Tomo I. Resultados Básicos. Madrid: Instituto Nacional de Estadística; 1995.

2. Wissler RW, Hiltscher L y Wahden M. Evolución precoz de las lesiores de las arterias coronarias en jóvenes: de la estría grasa a la placa fibrosa. Implicaciones para la :maginología. En: Fuster V, ed. Síndromes de Aterosclerosis. Correlaciones entre la imaginología, clínica y patología. Serie Monográfica de la American Heart Asociation. Barcelona: Medical Trends; 1993.p. 44-53.

3. Kannel WF. Factores de riesgo de coronariopatía: actualización del estudio de Framingham. Hosp Practice (ed. esp.)1991; 6: 45-55.

4. Banegas JR, Villar F, Martín-Moreno JM, Rodriguez Artalejo F, González J. Relevancia de la mortalidad por enfermedades del aparato circulatorio en España. Rev Clin Esp 1992; 190: 321-327.

5. Kannel WB. Clinical missconceptios dispelled by epidemiological research. Circulation 1995; 92 : 3.350-3.360.

6. García-Moll X, Badimón L. Biología molecular de la aterosclerosis. Medicine 1996: 22: 867-871. 
7. Scandinavian Simvastatin Survival Study Group: Randomised trial of cholesterol Lowering in 4444 patients with coronary heart disease: The Scandinavian Simvastatin Survival Study (4S). Lancet 1994; 344: 1.383-1.389.

8. Holme I. Relation of coronary heart disease incidence and total mortality to plasma cholesterol reduction in randomised trials: use of meta-analysis. Br Heart J 1993; 42-47.

9. Freedman DS, Srinivasan SR, Cresanta JL, Weber LS, Berenson GS. Cardiovascular risk factors from birth to 7 years of age: The Bogalusa Heart Study. Serum lipids and lipoproteins. Pediatrics 1987; 80: 789-796.

10. Boulton TJC, Magarey A, Cockington R. Colesterol desde la infancia hasta los 13 años: «tracking» y asociaciones padres-hijos. Anales Nestlé 1990; 48: 90-98.

11. Ruiz Moreno M, Gutierrez MT, Rincón P, Alvarez-Sala L, Camps MT. Hipercolesterolemia moderada en niños. ¿Indice de patología familiar? An Esp Pediatr 1986; 25: 322-328.

12. Sánchez M, González A, García B, Santos M, Serna C, Arias MA et al. Patrón lipídico de niños y adolescentes de Madrid. An Esp Pediatr 1992; 37: $205-210$.

13. Elcarte R, Villa I, Sada J, Gasco M, Oyarzabal $\mathrm{M}$, Sola A et al. Estudio de Navarra (PECNA). Hiperlipidemias II. Variaciones de los niveles medios de colesterol total, LDL-colesterol y triglicéridos de una población infanto-juvenil según edad y sexo. An Esp Pediatr 1993; 38: 159-166.

14. Rubio MJ, Tolosa M, Bellod P, Mariño M, De Oya M. Lipemia en relación a la edad. An Med Inter $1985 ; 5:$ 435-437.

15. Sarriá A, Ramos FJ, Bueno G, Puzo J, Giner A, Bueno M. Relaciones entre las tasas plasmáticas de colesterol y parámetros nutricionales, antropométricos, bioquímicos y hematológicos en escolares aragoneses. Rev Esp Pediatr 1988; 44: 241 247.

16. Plaza Perez I. y grupos de expertos de las sociedades españolas de Arteriosclerosis, Cardiología, Pediatría, Nutrición y Medicina Preventiva. Informe sobre el colesterol en niños y adolescentes españoles. An Esp Pediatr 1991; 35: 169-175.

17. Muñoz Calvo MT. Factores de riesgo cardiovascular en la infancia y adolescencia. [Tesis Doctoral]. Madrid: Universidad Autónoma; 1991.

18. López D, Plaza I, Muñoz MT, Madero R, Otero J, Hidalgo I, et al. Estudio de Fuenlabrada: Lípidos y lipoproteínas en niños y adolescentes. An Esp Pediatr 1989; 3: 342-349.
19. Sánchez M, Baeza J, Vila S, Asensio J, Arnaiz P, Ruiz-Jarabo C. Prevalencia de alteraciones lipídicas en niños y adolescentes de Madrid. Clin Invest Arterioscl 1992; 4: 114-118.

20. Comisión de lípidos y lipoproteínas de la Sociedad Española de Química Clínica. Protocolo para la obtención de especímenes en las determinaciones de lípidos y lipoproteínas. Quim Clin 1989; 8: 349-351.

21. Siedel J, Hagele D, Ziegenhorn J, Wahlefeld AE. Reagent for the enzymatic determination of serum total cholesterol with improved lipolytic efficiency. Clin Chem 1983; 29: 1.075-1.080.

22. Assman G, Schriewer H, Schmitz G, Hagele D. Quantification of high density lipoprotein cholesterol by precipitation with phosphotungstic acid and $\mathrm{Mg} \mathrm{C} 2$. Clin Chem 1983; 29: 2.026-2.030.

23. Friedewald WT, Levy RI, Fredrickson DS. Estimation of the concentration of C-LDL in plasma without use of the preparative ultracentrifuge. Clin Chem 1972; 18: 499-502.

24. Riepponen P, Marniemi J, Rautaoja T. Inmunoturbidimetric dertermination of apolipoproteins $\mathrm{AI}$ and B in serum. Scand J Clin Lab Invest 1987; 47: $739-744$

25. Consensus Conference. Lowering blood cholesterol to prevent heart disease. JAMA 1985; 253: 2.080-2.086.

26. Study Group European Atherosclerosis Society. The recognition and management of hyperlipidemia in adults: A policy statement of the European Atherosclerosis Society. Eur Heart J 1988; 9: 571 600 .

27. Carmena R, Ros E, Gómez JA. Recomendaciones para la prevención de la arteriosclerosis en España. Clin Invest Arteriosclerosis 1989; 1: 1-9.

28. Elcarte R, Villa I, Sada J, Gasco M, Oyarzabal M, Sola A, et al. Estudio de Navarra (PECNA). Hiperlipidemias V. ¿Cuál es la mejor definición de hiperlipidemia en la edad infanto-juvenil? An Esp Pediatr 1993; 38: 317-322.

29. Carrasco JL. El método estadístico en la investigación médica, 6. ${ }^{\mathrm{a}}$ ed. Madrid: Ciencia 3. 1995.

30. Blackburn H. Conference on the health effects of blood lipids: Optimal distributions for populations. Workshop report: Epidemiological section. Prev Med 1979; 8: 612-678.

31. Ministerio de Sanidad y Consumo. Indicadores de Salud, 2. ${ }^{a}$ evaluación en España del programa regional europeo «Salud para todos». Madrid: Ministerio de Sanidad y Consumo; 1993. 
32. Jimeno A, Giménez R, Vázquez JA, Rueda C. Estudio de la prevalencia de hipercolesterolemia en Extremadura. Rev San Hig Pública 1993; 67: 267-278.

33. National Cholestcrol Education Program. Report of the Expert Panel on Blood Cholesterol Levels in Children and Adolescents. Pediatrics 1992; 89: 525-584.

34. Muñoz Calvo MT. tratamiento dietético de las hipercolesterolemias. En: Hernández Rodriguez M, ed. Alimentación infantil, 2. ${ }^{a}$ ed. Madrid: Díaz Santos; 1993.p. 208-210.

35. Elcarte R, Villa I, Sada J, Gasco M, Oyarzabal $\mathrm{M}$, Sola A et al. Prevalencia de hipertensión arterial, hiperlipidemia y obesidad en la población infanto-juvenil de Navarra. Asociación de estos factores de riesgo. An Esp Pediatr 1993; 38: 428436.

36. Santos Tapia M, González A, García B, Serna C, Arias MA, Arnaiz P et al. Relación entre el perfil lipídico y la dieta en niños y adolescentes. Hipertensión y Arteriosclerosis 1991; 3: 24-29.

37. López T, Estivariz C. Martínez P, Jaunsolo MA, del Olmo D, Vázquez C y Grupo CAENPE. Consumo de alimentos del grupo «dulces y golosinas» en la población infantil escolarizada de la Comunidad Autónoma de Madrid. Med Clin (Barc) 1997; 109: 88-91.

38. Ministerio de Sanidad y Consumo. Consenso para el control de la Colesterolemia en España. Madrid: Ministerio de Sanidad y Consumo; 1989.

39. Bachorik PS. La medición del HDL-colesterol. Cardiovascular Risk Factors 1993; 2: 18-19.

40. Havel RJ, Rapaport E, Management of primary hyperlipidemia. N Engl J Med 1995; 332: 1.4911.498 .

41. Vella JC. Lípidos, lipoproteínas y apolipoprotcínas en sueros normales y en sueros hiperlipémicos. Análisis Clínicos 1990: XV: 141-146.

42. Vella JC. Parámetros lipoproteicos y enfermedad coronaria. Análisis Clínicos 1989; XIV: 283-287.

43. The Apoprotein and Antibody Standardization Program Planning Committee. The Apoprotein and Antibody Standardization Program. Arteriosclerosis 1989; 9: 144-145.
44. Dennison BA, Kikuchi DA, Srinivasan SR. Weber LS, Berenson GS. Measurement of apolipoprotein $\mathrm{B}$ as a screening test for identifying children with elevated levels of low-density lipoprotein choles terol. J Pediatr 1990; 117: 358-363.

45. Skovb F, Micic S, Jepsen B, Larsen SO, Hansen $B$, Teglund L ct al. Screening for familial hypercholesterolaemia by measurement of apolipoproteins in capillary blood. Arch Dis Child 1991; 66: 844-847.

46. Castelli WP, Abbot RC, McNamara PM. Summary estimates of cholesterol used to predict coronary heart disease. Circulation 1983; 67: 730-734.

47. Sociedad Española de Medicina Familiar y Comunitaria. Dislipemias. Manejo de las dislipemias en Aten Primaria. Barcelona: EMISA; 1997.p. 81.

48. Assmann G, Schulte H. Relation of high-density lipoprotein cholesterol and triglycerides to incidence of atherosclerosis coronary artery disease (the PROCAM experience). Am J Cardiol 1992; 70: 733-737.

49. Kannel WB. Low high-density lipoprotein cholesterol and what to do about it. Am J Cardiol 1992; 70: $810-814$.

50. Summary of the Second Report of the National Cholesterol Education Program Expert Panel on Detection. Evaluation and Treatment of High Blood Cholesterol in Adults (Adult Treatment Panel II) JAMA 1993; 269: 3.015-3.023

51. National Cholesterol Education Program. Report of the expert panel on detection, evaluation and treatment of high blood cholcstcrol in adults. Washington DC: Government Printing Office. 1988, NIH Publication 88-2925.

52. Sánchez M, Fernández ML. Colesterol HDL en la infancia. Su influencia en el diagnóstico de hipercolesterolemia. An Esp Pediatr 1997; 47: 285-288.

53. Elcarte R, Villa I, Sada J, Gasco M, Oyarzabal M, Sola A, et al. Estudio de Navarra (PECNA). Hiperlipidemias IV. Prevalencia de Hiperlipidemias en la población infanto-juvenil de Navarra. Variaciones según edad, sexo y Areas Sanitarias. An Esp Pediatr 1993; 38: 205-212.

54. Villar F, Banegas JR. La mortalidad cardiovascular en España. Rev San Hig Pública 1991; 65: $5-7$. 